\title{
On the Homological Category of 3-Manifolds
}

\author{
José CARLOS GÓMEZ LARRAÑAGA* y \\ FRANCISCO JAVIER GONZÁLEZ ACUÑ
}

\begin{abstract}
Let $M$ be a closed, connected, orientable 3-manifold. Denote by $n\left(S^{1} \times S^{2}\right)$ the connected sum of $n$ copies of $S^{1} \times S^{2}$. We prove that if the homological category of $M$ is three then for some $n \geq 1, H^{*}(M)$ is isomorphic (as a ring) to $H^{*}\left(n\left(S^{\prime} \times S^{2}\right)\right)$.
\end{abstract}

Let $X$ be a topological space. The (complete) homological category of $X$ [1] is the smallest cardinal $n$ such that there is a family $\mathscr{F}$ of open sets of $X$ with the following properties:

i) $\cup_{U \in}, U=X$.

ii) The cardinality of $n$ is $n$.

iii) For every $U \in \mathscr{F}, i>0$ and every ring of coefficients $R$, the inclusion induced homomorphism $H_{i}(U ; R) \rightarrow H_{i}(X ; R)$, in singular homology is zero.

We denote by cath $X$ the homological category of $X$. Let $M$ be a closed, connected, orientable 3-manifold. From the existence of a Heegaard splitting of $M$, it is clear that cath $M \leq 4$. Also, cath $M=2$ if only if $M$ is a homology 3-sphere [1]. Therefore, it suffices to characterize 3-manifolds $M$ with cath $M=3$. Denote by $n\left(S^{\perp} \times S^{2}\right)$ the connected sum of $n$ copies of $S^{t} \times S^{2}$. We will prove in Theorem I that if cath $M=3$ then for some $n \geq 1, H^{*}(M)$ is isomorphic (as a ring) to $H^{*}\left(n\left(S^{\mathrm{t}} \times S^{2}\right)\right)$. We work in the PL-category.

* Supported by the Heinrich Hertz Stiftung

1991 Mathematics Subject Classification: 57N10, 55M 30.

Editorial de la Universidad Complutense. Madrid, 1991. 


\section{I. . PRELIMINARIES}

Let $A$ be a finitely generated abelian group $A$. From now on, we will denote by tor $A$ (resp. rank $A$ ) the torsion subgroup of $A$ (resp. the number of summands $Z$ in $A / \operatorname{tor} A$ ).

The following lemma is used in the proof of Theorem 1. We feel it has independent interest; it can be used, for example, to calculate the homology of the complement of a 1-complex $K$ in a closed orientable 3 -manifold in terms of the cokernel of $H_{1}(K) \rightarrow H_{1}(M)$.

Lemma 1. Let $K$ be a compact proper 3-submanifold of the closed. connected and orientable 3-manifold $M$. Denote by $b_{i}\left(\right.$ resp. $\left.b_{i}^{\prime}\right)$ the $i$-th Betti number of $K$ (resp. $M-K)$. Then

$$
H_{1}(M-K) \oplus Z^{h_{2}} \approx C \mathcal{C}^{h_{1}+h_{n}-1}
$$

where $C$ is the cokernel of the inclusion induced homomorphism $H_{\mathrm{t}}(K) \rightarrow H_{1}(M)$. In particular, tor $H_{1}(M-K) \approx$ lor $C$.

Remark. The conclusion can also be stated as

$$
H_{1}(M-K) \approx C \oplus Z^{h_{11}+h_{0}^{\prime}-1-1 / 2(X(\partial K))}
$$

where, in case $m<0, \zeta \oplus Z^{m}$ is interpreted as the unique group $A$ such that $A \oplus Z^{-m} \approx \zeta$.

Proof. We have tor $\left(H_{1}(M-K) \oplus Z^{h_{2}}\right) \approx$ tor $H_{1}(M-K) \approx$ tor $H^{2}(M, K) \approx$ $\approx$ tor $H_{1}(M, K) \approx$ tor $C \approx$ tor $\left(C \oplus Z^{h_{1}+h_{i}-1}\right)$. The penultimate isomorphism being a consequence of the exact sequence

$$
H_{1}(K) \rightarrow H_{1}(M) \rightarrow H_{1}(M . K) \rightarrow H_{0}(K)
$$

where $H_{0}(K)$ is free abelian.

Also $b_{1}^{\prime}=\operatorname{rank} H_{1}(M \cdot K)=\operatorname{rank} H^{2}(M, K)=\operatorname{rank} H_{2}(M, K)$.

rank $H_{2}(M)=$ rank $H_{1}(M)$, rank $H_{3}(M, K)=b_{11}^{\prime}$ and the alternating sum of the ranks of the groups in the exact sequence

$$
\begin{aligned}
0 \rightarrow H_{3}(M) \rightarrow H_{3}(M, K) & \rightarrow H_{2}(K) \rightarrow H_{2}(M) \rightarrow H_{2}(M, K) \rightarrow H_{1}(K)- \\
& \rightarrow H_{1}(M) \rightarrow \zeta \rightarrow 0
\end{aligned}
$$


is zero. This yields $1-b_{0}^{\prime}+b_{2}+b_{1}-b_{1}-$ rank $C=0$ and, therefore, rank $\left(H_{1}(M-K) \oplus Z^{h_{2}}\right)=b_{1}^{\prime}+b_{2}=\operatorname{rank} C_{\zeta}+b_{1}+b_{0}^{\prime}-1=$

$$
=\operatorname{rank}\left(C \oplus Z^{h_{1}+h_{0}^{\prime}-1}\right) \text {. }
$$

Hence $H_{1}(M-K) \oplus Z^{h_{2}} \approx C € \oplus Z^{h_{1}+h_{n}-1}$. This completes the proof.

The following lemma is known $([4]$, p. 173, Corollarie V.8).

Lemma 2. Let W be a compact, orientable 3-manifold with $H_{1}(\partial W) \approx Z^{2 g}$. Then, the image of the inclusion induced homomorphism $H_{1}(\partial W) \rightarrow H_{1}(W)$ has rank $\mathrm{g}$.

Let $M$ be a closed $n$-manifold. We follow [3] for the definition and properties of an $n$-filling of $M$.

Lemma 3. Let $\left\{T_{i}\right\}_{i=1}^{3}$ be a 3-filling of the closed. connected and orientable 3-manifold $M$. Let $W$ be a regular neighbourhood of $F=\cup{ }_{i=1}^{3} \partial T_{i}$. Then, the image of $H_{1}(\partial W) \rightarrow H_{1}(W)$ contains tor $H_{1}(W)$.

Proof. Let $F_{k}=T_{i} \cap T_{j}$, where $\{i, j, k\}=\{1,2,3\}$. Let $C_{i}$ be a product neighbourhood of $\partial T_{i}$ in $T_{i}$. We may assume $W=\bigcup_{i=1}^{3} C_{i}$. Write $\partial_{i} W=C_{i} \cap \partial W$. We have the commutative diagram

$$
\begin{aligned}
& H_{1}\left(\partial T_{1}\right)-H_{1}(F) \rightarrow H_{1}\left(F, \partial T_{1}\right) \\
& \downarrow \\
& \begin{array}{c}
H_{1}\left(C_{1}\right) \\
\uparrow
\end{array} \mid \\
& H_{1}\left(\partial_{1} W\right)-H_{1}(W)
\end{aligned}
$$

where the upper row is exact and the vertical arrows are isomorphism. Since $H_{1}\left(F_{,} \partial T_{1}\right) \approx H_{1}\left(F_{1}, \partial F_{1}\right) \approx H^{\prime}\left(F_{1}\right)$ is free, the image of $H_{1}\left(\partial T_{1}\right) \rightarrow H_{1}(F)$ contains tor $H_{1}(F)$ and, therefore, the image of $H_{1}\left(\partial_{1} W\right) \rightarrow H_{1}(W)$ contains tor $H_{1}(W)$ from which the result follows.

\section{MAIN RESULT}

Theorem 1. Let $M$ be a closed, connected, orientable 3-manifold. 'If' ath $M=3$ then for some $n \geq 1, H^{*}(M)$ is isomorphic (as a ring) to $H^{*}\left(n\left(S^{1} \times S^{2}\right)\right)$. 
Proof. By the arguments of [3] (see also [2]), there exists a 3-filling $\left\{T_{i}\right\}_{i=1}^{3}$ of $M$, where $T_{i}$ is a cube with handles and $H_{1}\left(T_{i}\right) \rightarrow H_{1}(M)$ is trivial for $i=1,2,3$. Let $W$ be a regular neighbourhood of $U_{i=1}^{3} \partial T_{i}$ in $M$ and let $K$ be a closure of $M-W$. Thus $K$ is a disjoint union of three cubes with handles $\left\{K_{i}\right\}_{i=1}^{3}$. Let $g_{i}$ be the genus of $\partial K_{i}$ and $g=g_{1}+g_{2}+g_{3}$. Consider the commutative diagram with exact rows.

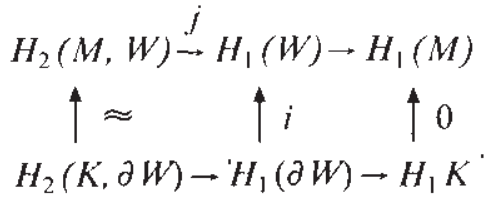

From the fact that the right vertical homomorphism is trivial we obtain $\operatorname{Im} i \subset \operatorname{Im} j$, and, since the left vertical homomorphism is onto, $\operatorname{Im} j \subset \operatorname{Im} i$. Hence $\operatorname{Im} j=\operatorname{Im} i$, which by lemmas 2 and 3 , is isomorphic to $Z^{g} \oplus$ tor $H_{1}(W)$. Since $H_{2}(M, W) \approx H^{\prime}(K) \approx Z^{k}$, Im $j$ can be generated by $g$ elements and, therefore, we must have tor $H_{1}(W)=0$. By lemma 1, it follows that tor $H_{1} M \approx$ tor $H_{1}(W)=0$ so that $H_{1} M$ is free abelian. The rank $n$ of $H_{1} M$ must be positive since, otherwise, cath $M$ would be two. Hence $H^{i}(M) \approx Z^{n}$ for $i=1,2$, that is the cohomology of $M$ is additively the same as that of $n\left(S^{1} \times S^{2}\right)$.

Let $\left\{a_{1}, \ldots, a_{n}\right\}$ be a basis of $H^{1}(M)$ and let $\left\{b_{1}, \ldots, b_{n}\right\} \subset H^{2}(M)$ be the dual basis; that is $a_{i}-b_{j}=\delta_{i j} \nu$ where $\nu \in H^{3}(M)$ is the fundamental class. For $r=1,2,3, H^{\prime}(M) \rightarrow H^{\prime}\left(T_{r}\right)$ is trivial so that $a_{i}$ is the image of an element $a_{i}^{(r)} \in H^{l}\left(M, T_{r}\right)$ under $H^{\mathrm{t}}\left(M, T_{r}\right) \rightarrow H^{\mathrm{l}}(M)$. Then, for any $i, j, k, a_{i}-a_{j}-a_{k}$ is zero since it is the image, in $H^{3}(M)$, of $a_{i}^{(1)} \sim a_{j}^{(2)} \cup a_{k}^{(3)} \in H^{3}\left(M, T_{1} \cup T_{2} \cup T_{3}\right)$ $=0$.

Moreover, for any $i, j, a_{i}-a_{j}=0$ because, if we write $a_{i}-a_{i}=\sum_{k=1}^{n} n_{k} b_{k}$, then $0=a_{k} \backsim a_{i} \backsim a_{j}=n_{k} \nu$ so that $n_{k}=0$ for $k=1, \ldots, n$. This proves that the cohomology ring of $M$ is isomorphic to that of $n\left(S^{1} \times S^{2}\right)$. This completes the proof of the theorem.

Aknowledgement. The first author wishes to thank the Ruhr-Universität Bochum for support and hopitality during the preparation of this report. 


\section{References}

[1] Fox, R. H,: “On the l-usternik-Schnirelmann category", Amm. Math, 42 (1941). 333-370.

[2] MON'TE:IANO, L.: «A quick proof of Singhof's cat $\left(M \times S^{1}\right)=$ cat $(M)+1$ theorem" Manuscripta Math. 42 (1989), 49-52.

[3] Singhol-, W.: "Minimal coverings of manifolds with balls", Manuscripra Math. 29 (1979). 385-415.

[4] Thom, R.: «Espaces fibrés en spheress et carrés de Steenrodm. Amn. Sci. Ecole Nom. Sup. (3) 69 (1952), 109-182.

INSTITUTO DEE MATEMATICAS

Universidad Nacional Autonomat de México

Ciudad Universitariat

04510 México. D. F

México.

Recibida: 3 de septiembre de 1990 